\title{
SISTEMA ORIGINAL DE ARCHIVO EN CONSULTORIOS PRIVADOS
}

\section{Dr. Saulo Muñoz Delgado*}

La enseñanza de nuestras escuelas médicas y el ejercicio de la profesión están fundamentalmente orientadas hacia la práctica privada, o sea una medicina de consultorio y terapia. Es tan cierto esto que en la realidad nacional casi nadie se aparta de esta tendencia y hasta los grupos dedicados a la investigación más sofisticada, ya sea por la situación económica de las Instituciones o por razones personales, tienden a hacer el mayor ejercicio profesional que les permiten sus actividades.

A pesar de estas consideraciones y de que en los estudios se han establecido clases de estadísticas, no se les enseña a llevar un archivo que puede ser la infraestructura más importante en el ejercicio profesional.
Tampoco en los Congresos Médicos se han presentado trabajos de este orden y aún en las revistas dedicadas a temas médicos prácticos no es posible encontrar un artículo sobre este tópico.

Teniendo en cuenta que la mayoría de las personas que manejan los archivos en consultorios son empíricas, es todavía más urgente organizar un sistema fácil y práctico pues el historial médico es fundamental en la práctica médica. Un método adecuado, fácil y correcto en el manejo de las historias, nos deriva sinnúmero de ventajas en la atención de los consultorios tanto en el orden técnico como en economía y utilización del tiempo.

* Jefe Dpto. de Obst. y Ginec. ICSS. Valle.

CUADRO № 1

RECORD DE PACIENTES ATENDIDAS DURANTE EL 1er. SEMESTRE DEL AÑO 1975

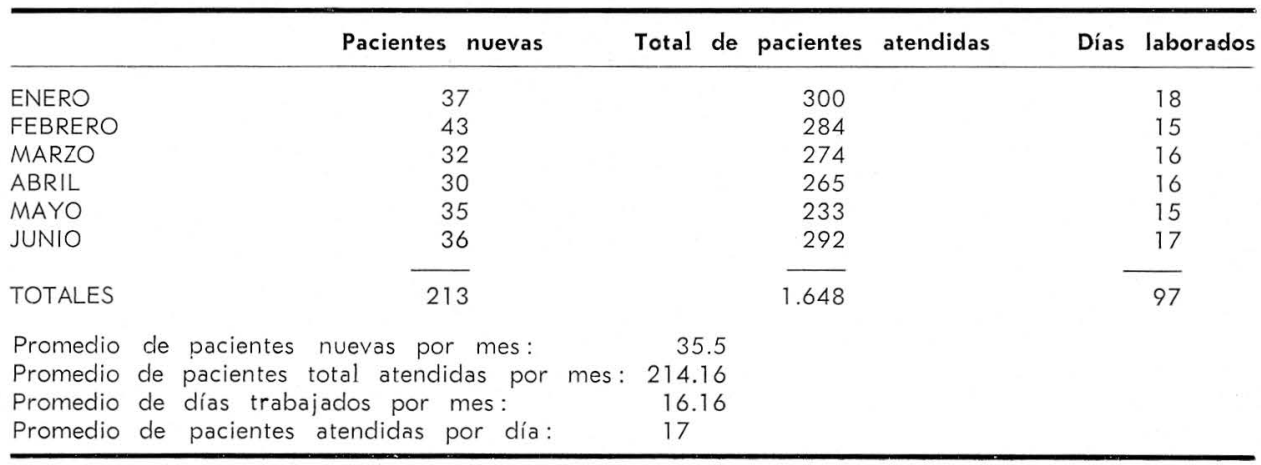


CUADRO N: 2

RECORD DE PACIENTES ATENDIDAS DURANTE EL 2\% SEMESTRE DEL AÑO 1974

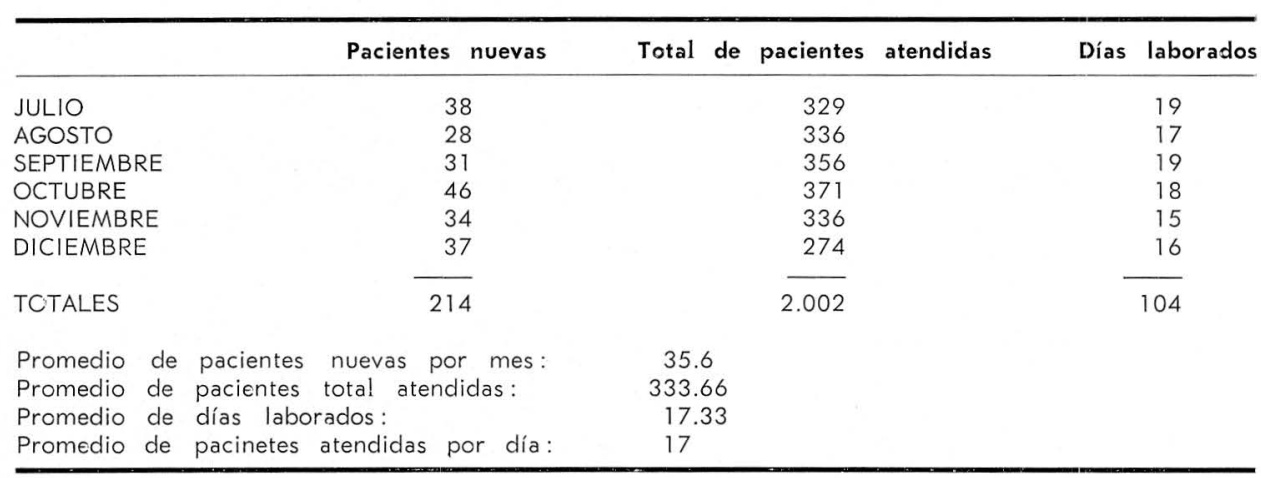

CUADRO № 3

NUMERO DE SUSCRIPTORES DEL DIRECTORIO TELEFONICO DE CALI 1975

\begin{tabular}{|c|c|c|c|c|c|}
\hline & Letra & Número de hojas & Número de pág. & Suscrip. por pág. & Total \\
\hline 1 & c & 15 & 31 & 280 & 8.680 \\
\hline 2 & A & $12 \frac{1}{2}$ & 23 & " & 6.446 \\
\hline 3 & M & 8 & 17 & " & 4.960 \\
\hline 4 & G & $71 / 2$ & 15 & " & 4.880 \\
\hline 5 & $\mathrm{R}$ & 7 & 14 & $"$ & 3.920 \\
\hline 6 & s & 7 & 14 & " & 3.920 \\
\hline 7 & 6 & $6 \frac{1 / 2}{2}$ & 13 & $"$ & 3.640 \\
\hline 8 & B & 6 & 12 & $"$ & 3.360 \\
\hline 9 & $\mathrm{~F}$ & $5 \frac{1}{2}$ & 11 & $"$ & 3.080 \\
\hline 10 & T & $5 \frac{1 / 2}{2}$ & 11 & $"$ & 3.080 \\
\hline 11 & L & 5 & 10 & $"$ & 2.800 \\
\hline 12 & $\mathrm{v}$ & 5 & 10 & $"$ & 2.800 \\
\hline 13 & D & $4 \frac{1}{2}$ & 9 & $"$ & 2.520 \\
\hline 14 & E & $4 \frac{1}{2}$ & 9 & " & 2.520 \\
\hline 15 & 1 & $31 / 2$ & 7 & $"$ & 1.960 \\
\hline 16 & $\mathrm{H}$ & 3 & 6 & $"$ & 1.680 \\
\hline 17 & 0 & $2 \frac{1}{2}$ & 5 & $"$ & 1.400 \\
\hline 18 & J & 2 & 4 & $"$ & 1.120 \\
\hline 19 & K & 1 & 2 & $"$ & 560 \\
\hline 20 & $\mathrm{~N}$ & 1 & 2 & $"$ & 560 \\
\hline 21 & u & 1 & 2 & $"$ & 560 \\
\hline 22 & Q & 1 & 2 & $"$ & 560 \\
\hline 23 & Z & 1 & 2 & $"$ & 560 \\
\hline 24 & LL & $1 / 2$ & 1 & $"$ & 280 \\
\hline 25 & $\mathrm{CH}$ & $1 / 2$ & 1 & $"$ & 280 \\
\hline 26 & W & & $1 / 4$ & & \\
\hline 27 & Y & & $1 / 4$ & & \\
\hline 28 & $\tilde{N}$ & & & 2 suscriptores & \\
\hline 29 & $x$ & & & 2 suscriptores & \\
\hline
\end{tabular}




\section{Material y Métodos}

Partimos de la base de un consultorio, atendido durante 6 u 8 horas diarias, con una práctica intensa y con un término de tiempo de ejercicio de la profesión de aproximadamente 30 años.

De acuerdo al cálculo de pacientes que se puede ver en el transcurso de la vida, dejamos un margen de 4-6 veces mayor, con el fin de que en un futuro este número de profesionales pueden asociarse o continuar con el mismo archivo.

Se practica un cálculo para la población de la ciudad con el número de personas inscritas en cada letra del índice del Directorio telefónico de la ciudad, cuyo número de suscriptores en Cali es de más o menos $80 \mathrm{mil}$ o 90 mil personas.

\section{CUADRO NN 4}

\section{INCIDENCIA DE SUSCRIPTORES}

POR GRUPOS DE 7.000 a $\mathbf{5 0 0}$

\begin{tabular}{|c|c|c|c|c|c|c|}
\hline LETRAS & $\mathrm{DE}$ & MAS & DE & 7.000 & $=$ & 1 \\
\hline LETRAS & DE & MAS & $\mathrm{DE}$ & 5.000 & $=$ & 1 \\
\hline LETRAS & DE & MAS & $\mathrm{DE}$ & 4.000 & $=$ & 2 \\
\hline LETRAS & DE & MAS & DE & 3.000 & $=$ & 6 \\
\hline LETRAS & DE & MAS & DE & 2.000 & $=$ & 4 \\
\hline LETRAS & $\mathrm{DE}$ & MAS & DE & 1.000 & $=$ & 4 \\
\hline LETRAS & DE & MAS & DE & 500 & $=$ & 5 \\
\hline LETRAS & DE & MENOS & $\mathrm{DE}$ & 500 & $=$ & 6 \\
\hline & & & & & & 29 \\
\hline
\end{tabular}

Tomamos de esta manera 100.000 números, lo dividimos por 29 letras, dando un promedio de 3.500 para cada letra. De acuerdo a las necesidades las distribuímos según el Cuadro No 5. Después establecemos una guía para inscribir las pacientes a medida que ingresan al consultorio.

\section{CUADRO NN 5}

\section{DISTRIBUCION DE LAS LETRAS POR INCIDENCIA}

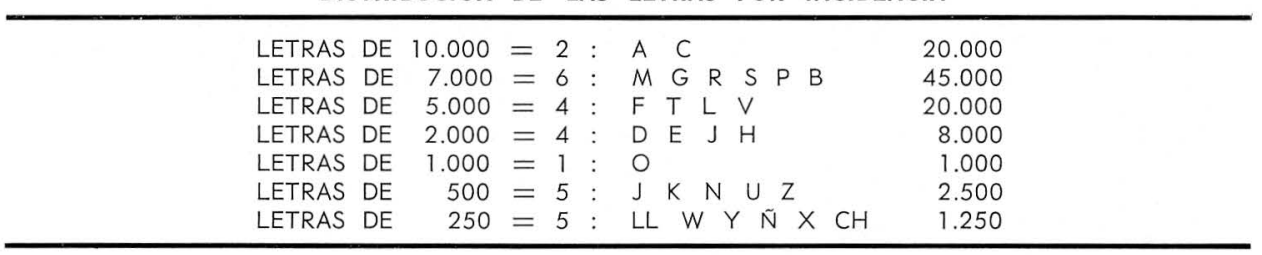

\section{CUADRO N: 6}

DISTRIBUCION DE NUMEROS DE ACUERDO AL ORDEN ALFABETICO

\begin{tabular}{|c|c|c|c|c|c|c|c|}
\hline \multicolumn{4}{|c|}{ ORDINAL } & \multicolumn{4}{|c|}{ ORDINAL } \\
\hline A & 00.001 & - & 9.999 & $N$ & 64.000 & - & 64.499 \\
\hline B & 10.000 & - & 17.499 & $\tilde{N}$ & 64.500 & - & 64.749 \\
\hline C & 17.500 & - & 27.499 & O & 64.750 & - & 65.749 \\
\hline $\mathrm{CH}$ & 27.500 & - & 27.749 & $P$ & 65.750 & - & 73.249 \\
\hline $\mathrm{D}$ & 27.750 & - & 30.249 & $Q$ & 73.250 & - & 73.499 \\
\hline$E$ & 30.250 & - & 32.749 & $\mathrm{R}$ & 73.500 & - & 80.999 \\
\hline$F$ & 32.750 & - & 37.749 & $S$ & 81.000 & - & 88.499 \\
\hline G & 37.750 & - & 45.249 & $\mathrm{~T}$ & 88.500 & - & 93.499 \\
\hline$H$ & 45.250 & - & 47.749 & $U$ & 93.500 & 一 & 93.999 \\
\hline 1 & 47.750 & - & 50.249 & V & 94.000 & - & 98.999 \\
\hline J & 50.250 & - & 50.749 & W & 99.000 & - & 99.249 \\
\hline K & 50.750 & - & 51.249 & $x$ & 99.250 & - & 99.499 \\
\hline L & 51.250 & - & 56.249 & Y & 99.500 & - & 99.749 \\
\hline LL & 56.250 & - & 56.499 & Z & 99.750 & - & 100.000 \\
\hline$M$ & 56.500 & - & 63.999 & & & & \\
\hline
\end{tabular}



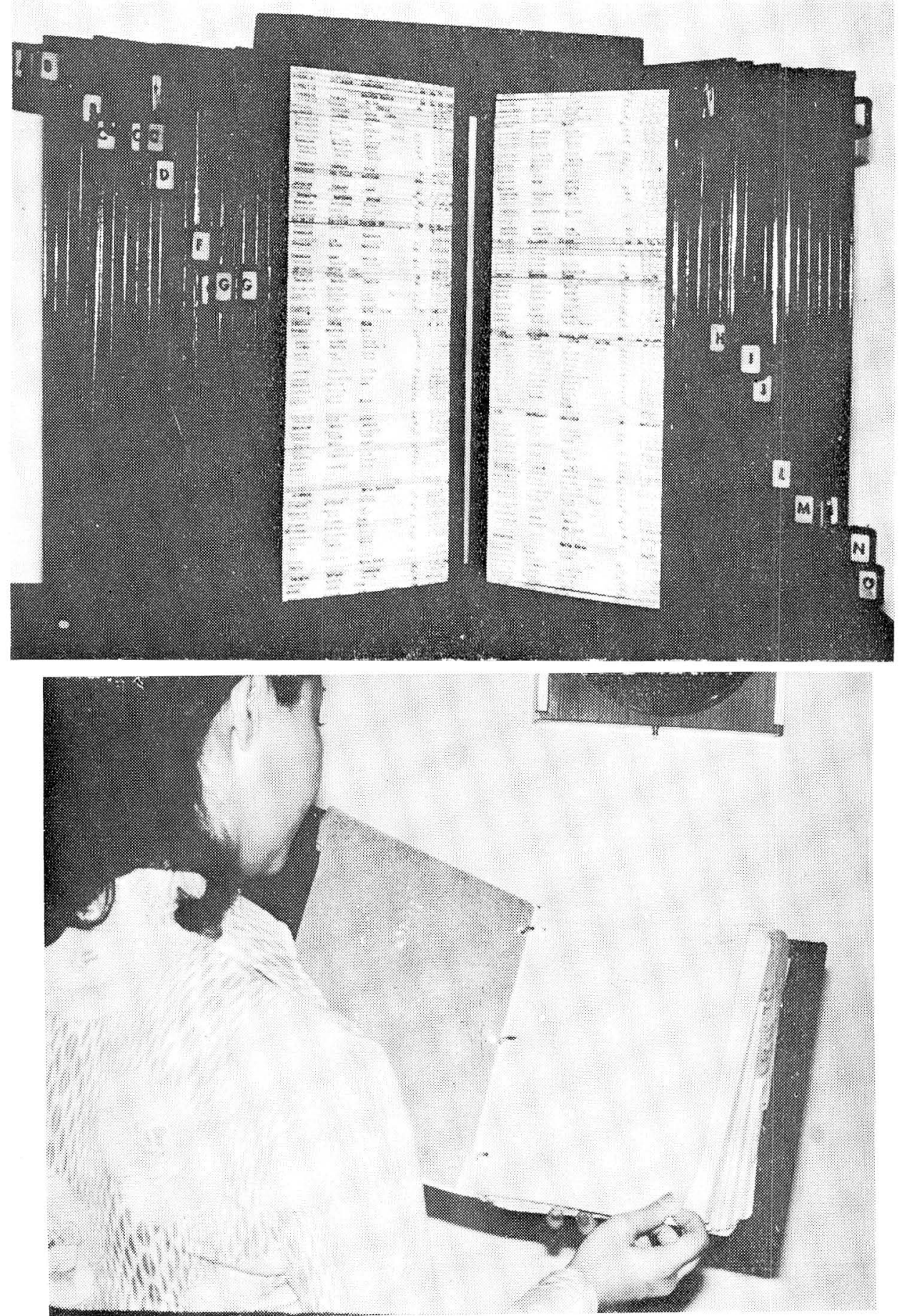


\section{Resultados}

1. Sistema simplificado del archivo, pues cada letra constituye por sí sola una parte, lo cual excluye el manejo de las otras 28 letras.

2. Cada paciente tiene una identificación por abecedario y queda dentro de un número limitado de la clasificación general, lo cual facilita el archivo de historias y disminuye el riesgo de extravío pues de base excluye las 28 letras restantes.

3. Esta fragmnetación del archivo por letras tiene las siguientes ventajas:

a) Reduce el riesgo de errores en el archivo de historias en $1 \times 28$.

b) En caso de extravío facilita el encontrar las historias primeramente por la identificación de cada letra y segundo por la exclusión de las 28 partes restantes del archivo.

c) Economía de tiempo por la rápida obtención, dada la fragmentación del archivo que disminuye en 28 partes su búsqueda.

d) Personal empírico puede manejar este sistema de archivo de adopción fácil en cualquier consultorio.

e) Es un sistema que permite el registro técnico durante toda la vida, para más o menos 6 médicos con una intensa y numerosa clientela.

f) Es un método aplicable a archivos de 100.000 historias, que representa una población de pacientes de una ciudad de aproximadamente un millón de habitantes.

g) Es aplicable en otros campos como hospitales, instituciones, pro- gramas de investigación, laboratorio, clínica de especialidad, programas especiales, etc.

h) En la misma forma del estudio presentado, haciendo cálculos en la población de los promedios de apellidos de cada ciudad, puede aplicarse a comunidades del orden del $\mathrm{Mi}$ llón o más.

\section{Conclusiones}

El sistema de archivo propuesto, en el curso de 20 años de práctica privada, ha demostrado ser eficiente para el manejo de una comunidad grande, economiza tiempo, y facilita la rápida obtención de historias, permite laborar estadísticas, responder con precisión llamaads telefónicas, hacer las anotaciones del caso, aumentar el número de pacientes en la consulta etc. Establece continuidad con otros médicos que lo reemplazan en el consultorio, y es manejable aun por personas empíricas en estas técnicas.

\section{Summary}

The file sistem propsed, during 20 years of private practice, has shown to be efficient for the management of a big comunity, it economize time, facilitates the rapid obtantion of histories, permits the statistics elaboration, to answer the phone calls pressicion, to make the needed annotations, to increased number of patients in the consult, etc. Stablish continuity with other Doctors who might replazed at the Doctor's Office, and is eassy for mange even though by empiric people in these techniques. 